TAIWANESE JOURNAL OF MATHEMATICS

Vol. 13, No. 1, pp. 47-65, February 2009

This paper is available online at http://www.tjm.nsysu.edu.tw/

\title{
STABILITY OF IMPLICIT MULTIFUNCTIONS IN ASPLUND SPACES
}

\author{
N. Q. Huy and J.-C. Yao*
}

\begin{abstract}
The purpose of this paper is to present new sufficient conditions for both the metric regularity and the Lipschitzian stability of implicit multifunctions in Asplund spaces. The basic tools of our analysis involve the Fréchet normal coderivative and the Mordukhovich normal coderivative of set-valued mappings, the basic subgradient estimate for marginal functions and the Ekeland variational principle. Applications to the pointbased characterizations for the metric regularity and the Lipschitzian stability of solution mapping of parametric generalized equations are given.
\end{abstract}

\section{INTRODUCTION}

Consider $F: X \times Y \rightrightarrows Z$ a set-valued mapping between Banach spaces and the generalized inequality systems

$$
0 \in F(x, y) .
$$

The solution map $G: Y \rightrightarrows X$ associated with (1.1) which has the form

$$
G(y)=\{x \in X: 0 \in F(x, y)\}
$$

is said to be the implicit multifunction defined by the inclusion (1.1). In this paper we will focus on the study of the metric regularity in the Robinson's sense [24] and the Lipschitzian stability in the Aubin's sense [1] for the implicit multifunction (1.2).

\footnotetext{
Accepted April 14, 2008.

Communicated by M. H. Shih.

2000 Mathematics Subject Classification: 49J52, 49J53, 90C31.

Key words and phrases: Implicit multifunction, Metric regularity in Robinson's sense, Lipschitzian stability in Aubin's sense, Mordukhovich normal coderivative, Fréchet coderivative, Ekeland variational principle.

This research was partially supported by the grant NSC 97-2115-M-110-001.

*Corresponding author.
} 
The study of implicit multifunction has a long history. Stable properties of implicit multifunction such as the metric regularity and Lipschitzian stability which have attracted much attention of many researchers; see, e.g., [2-19, 22-27] and the references therein for more details and discussions.

In a special case of $F$ in (1.1) which has the form

$$
F(x, y)=f(x, y)+Q(x, y)
$$

where $f: X \times Y \rightarrow Z$ is a single-valued mapping and $Q: X \times Y \rightrightarrows Z$ is a set-valued mapping between Asplund spaces, Mordukhovich [12, 14, 15] gave the characterizations of necessary as well as sufficient conditions for the Lipschitzian stability of (1.2) under the strict differentiability of $f$ with the surjective property of partial gradient of $f$ and either $Q$ does not depend $x$ or $Q$ is graphically regular at a given point. Together with the Lipschitzian stability, the metric regularity of (1.2) was intensively investigated in implicit and inverse multifunctions (see, e.g., [2-11, 13, 16-19, 22-27]). Recently, Ledyaev and Zhu [9], Ngai and Théra [22] established sufficient conditions for the metric regularity of (1.2) in terms of the Fréchet normal coderivatives in Banach space with the Fréchet-smooth bump function. Another set of sufficient conditions for the same property in Asplund spaces was given by Lee, Tam and Yen [10] in terms of the normal coderivatives. More recently, Yen and Yao [27] established some pointbased sufficient conditions for the metric regularity property of implicit multifunctions in finite-dimensional setting, and the same property in the WCG space setting was also presented in [7]. It is well known that for inverse multifunctions the metric regularity is equivalent to the Lipschitzlike property (see $[3,11,23]$ ). However, as well shown in [8, Example 3.6 and 3.7] that the metric regularity of implicit multifunctions is not equivalent to the Lipschitz-like property in general.

Our main objective of this paper is to establish new sufficient conditions for both the (local) metric regularity and Lipschitzian stability of the solution map (1.2) in Asplund space setting. The basic tools of our analysis involve the Fréchet normal coderivative and the Mordukhovich normal coderivative of set-valued mappings, the basic subgradient estimate for marginal functions and the Ekeland variational principle. Applications to the pointbased characterizations for both the metric regularity and the Lipschitzian stability of solution mapping of parametric generalized equations (1.1)-(1.3) are also given without any requirements of either the surjective property of partial gradient of $f, Q$ does not depend $x$ or $Q$ is graphically regular at a given point. Some our results generalized the corresponding results presented in $[7,10,27]$.

The paper is organized as follows. In Section 2 we recall some basic definitions and preliminaries from variational analysis and the generalized differentiation. In Section 3 we derive pointbased sufficient conditions for both the (local) metric 
regularity and Lipschitzian stability of the implicit multifunction (1.2), and the relationships with the corresponding known results. Applications to stability analysis for solution mapping of parametric generalized equations are given in Section 4.

\section{Preliminaries}

Throughout the paper we use standard notation of variational analysis and generalized differentiation. We refer the reader to the books by Mordukhovich [16, 17] for more details and discussions. Unless otherwise stated, all spaces under consideration are Banach spaces whose norms are always denoted by $\|\cdot\|$. For any $X$ we consider its dual space $X^{*}$ equipped with the weak* topology $w^{*}$ where $\langle\cdot, \cdot\rangle$ means the canonical pairing. As usual, $B_{X}$ and $B_{X^{*}}^{*}$ stand for the closed unit balls of the Banach space $X$ and its dual, respectively. The symbol $A^{*}$ is the adjoint operator of a linear continuous operator $A$. The closed ball with center $x$ and radius $\rho$ is denoted by $B_{\rho}(x)$.

Given a set-valued mapping $F: X \rightrightarrows X^{*}$ between a Banach space $X$ and its topological dual $X^{*}$, we denote by

$$
\begin{aligned}
\operatorname{Limsup}_{x \rightarrow \bar{x}} F(x):=\left\{x^{*} \in X^{*} \mid\right. & \exists \text { sequences } x_{k} \rightarrow \bar{x} \text { and } x_{k}^{*} \stackrel{w^{*}}{\longrightarrow} x^{*} \\
& \text { with } \left.x_{k}^{*} \in F\left(x_{k}\right) \text { for all } k \in \mathbb{N}\right\}
\end{aligned}
$$

the sequential Painleve-Kuratowski upper/outer limit with respect to the norm topology of $X$ and the weak ${ }^{*}$ topology of $X^{*}$, where $\mathbb{N}:=\{1,2, \ldots\}$.

Given $\Omega \subset X$ and $\varepsilon \geq 0$, define the collection of $\varepsilon$-normals to $\Omega$ at $\bar{x} \in \Omega$ by

$$
\widehat{N}_{\varepsilon}(\bar{x} ; \Omega):=\left\{x^{*} \in X^{*} \mid \limsup _{x \stackrel{\Omega}{\longrightarrow} \bar{x}} \frac{\left\langle x^{*}, x-\bar{x}\right\rangle}{\|x-\bar{x}\|} \leq \varepsilon\right\},
$$

where $x \stackrel{\Omega}{\rightarrow} \bar{x}$ means that $x \rightarrow \bar{x}$ with $x \in \Omega$. When $\varepsilon=0$, the set $\widehat{N}(\bar{x} ; \Omega):=$ $\widehat{N}_{0}(\bar{x} ; \Omega)$ in (2.1) is a cone called the prenormal cone or the Fréchet normal cone to $\Omega$ at $\bar{x}$.

The Mordukhovich normal cone $N(\bar{x} ; \Omega)$ is obtained from $\widehat{N}_{\varepsilon}(x ; \Omega)$ by taking the sequential Painlevé-Kuratowski upper limit in the weak* topology of $X^{*}$ as

$$
N(\bar{x} ; \Omega):=\operatorname{Limsup}_{\substack{x \\ \varepsilon \downarrow 0}} \widehat{N}_{\varepsilon}(x ; \Omega),
$$

where one can put $\varepsilon=0$ when $\Omega$ is closed around $\bar{x}$ and the space $X$ is Asplund, i.e., a Banach space whose separable subspaces have separable duals. The subset 
$\Omega \subset X$ is said to be (locally) closed around $\bar{x}$ if there is a neighborhood $U$ of $\bar{x}$ such that $\Omega \cap \mathrm{cl} U$ is closed.

Let $F: X \rightrightarrows Y$ be a set-valued mapping between Banach spaces with the graph

$$
\operatorname{gph} F:=\{(x, y) \in X \times Y \mid y \in F(x)\} .
$$

The Mordukhovich normal coderivative $D^{*} F(\bar{x}, \bar{y}): Y^{*} \rightrightarrows X^{*}$ of $F$ at $(\bar{x}, \bar{y}) \in$ gph $F$ is defined by

$$
D^{*} F(\bar{x}, \bar{y})\left(y^{*}\right):=\left\{x^{*} \in X^{*} \mid\left(x^{*},-y^{*}\right) \in N((\bar{x}, \bar{y}) ; \operatorname{gph} F)\right\} .
$$

The Fréchet normal coderivative at $(\bar{x}, \bar{y}) \in \operatorname{gph} F$ is defined by

$$
\left.\widehat{D}^{*} F(\bar{x}, \bar{y})\left(y^{*}\right):=\left\{x^{*} \in X^{*} \mid\left(x^{*},-y^{*}\right) \in \widehat{N}((\bar{x}, \bar{y}) ; \operatorname{gph} F)\right)\right\} \quad \forall y^{*} \in Y^{*} .
$$

A single-valued mapping $f: X \rightarrow Y$ is said to be strictly differentiable at $\bar{x}$ if there is a linear continuous operator $\nabla f(\bar{x}): X \rightarrow Y$ such that

$$
\lim _{x, u \rightarrow \bar{x}} \frac{f(x)-f(u)-\langle\nabla f(\bar{x}), x-u\rangle}{\|x-u\|}=0 .
$$

We known that for such mappings one has

$$
D^{*} f(\bar{x})\left(y^{*}\right)=\widehat{D}^{*} f(\bar{x})\left(y^{*}\right)=\left\{(\nabla f(\bar{x}))^{*} y^{*}\right\} \quad \forall y^{*} \in Y^{*},
$$

i.e., the Mordukhovich normal coderivative (resp., Fréchet normal coderivative) is a generalization of the adjoint operator to the classical Jacobian/strict derivative. For more details, we refer the reader to [16].

For an extended real-valued function $\varphi: X \rightarrow \overline{\mathbb{R}}:=[-\infty, \infty]$, we define

$$
\operatorname{dom} \varphi=\{x \in X|| \varphi(x) \mid<\infty\}, \quad \text { epi } \varphi=\{(x, \mu) \in X \times \mathbb{R} \mid \mu \geq \varphi(x)\},
$$

and say that $\varphi$ is lower semicontinuous at $\bar{x} \in X$ if $\liminf _{x \rightarrow \bar{x}} \varphi(x) \geq \varphi(\bar{x})$. Here $\liminf _{x \rightarrow \bar{x}}$ denotes the lower limit of scalar functions in the classical sense.

Each extended real-valued function $\varphi: X \rightarrow \overline{\mathbb{R}}$ corresponds to a multifunction $\Phi: X \rightrightarrows \mathbb{R}$ defined by

$$
\Phi(x)=E_{\varphi}(x):=\{\mu \in \mathbb{R} \mid \mu \geq \varphi(x)\} \quad \forall x \in X,
$$

which is called the associated epigraphical multifunction. The limiting or basic/Mordukhovich subdifferential $\partial \varphi(\bar{x})$ of $\varphi$ at $\bar{x} \in \operatorname{dom} \varphi$ is defined via the coderivative $D^{*} \Phi(\bar{x}, \bar{y})$ as follows

$$
\partial \varphi(\bar{x}):=D^{*} E_{\varphi}(\bar{x}, \varphi(\bar{x}))(1)=\left\{x^{*} \in X \mid\left(x^{*},-1\right) \in N((\bar{x}, \varphi(\bar{x})) ; \text { epi } \varphi)\right\} .
$$


If $\bar{x} \notin \operatorname{dom} \varphi$ then one puts $\partial \varphi(\bar{x})=\emptyset$. If $\bar{x}$ is a local minimum of $\varphi$, then

$$
(0,-1) \in \widehat{N}((\bar{x}, \varphi(\bar{x})) ; \operatorname{epi} \varphi) \subset N((\bar{x}, \varphi(\bar{x})) ; \operatorname{epi} \varphi) .
$$

The presubdifferential or Fréchet subdifferential of $\varphi$ at $\bar{x} \in \operatorname{dom} \varphi$ is denoted by

$$
\widehat{\partial} \varphi(\bar{x}):=\left\{x^{*} \in X \mid\left(x^{*},-1\right) \in \widehat{N}((\bar{x}, \varphi(\bar{x})) ; \text { epi } \varphi)\right\} .
$$

We recall that a set $\Omega$ is sequentially normally compact (SNC) at $\bar{x}$ if for any sequences $\varepsilon_{k} \downarrow 0, x_{k} \stackrel{\Omega}{\rightarrow} \bar{x}$, and $x_{k}^{*} \in \widehat{N}_{\varepsilon_{k}}\left(x_{k} ; \Omega\right)$ one has

$$
\left[x_{k}^{*} \stackrel{w^{*}}{\rightarrow} 0\right] \Longrightarrow\left[\left\|x_{k}^{*}\right\| \rightarrow 0\right] \text { as } k \rightarrow \infty
$$

where $\varepsilon_{k}$ can be omitted if $X$ is Asplund and if $\Omega$ is locally closed around $\bar{x}$. A set-valued mapping $F: X \rightrightarrows Y$ is $S N C$ at $(\bar{x}, \bar{y}) \in \operatorname{gph} F$ if its graph enjoys this property. $F$ is partially sequentially normally compact (PSNC) at $(\bar{x}, \bar{y})$ if for any sequence $\left(\varepsilon_{k}, x_{k}, y_{k}, x_{k}^{*}, y_{k}^{*}\right) \in[0, \infty) \times(\operatorname{gph} F) \times X^{*} \times Y^{*}$ satisfying

$$
\varepsilon_{k} \downarrow 0,\left(x_{k}, y_{k}\right) \rightarrow(\bar{x}, \bar{y}), x_{k}^{*} \in \widehat{D}_{\varepsilon_{k}}^{*} F\left(x_{k}, y_{k}\right)\left(y_{k}^{*}\right), x_{k}^{*} \stackrel{w^{*}}{\rightarrow} 0 \text { and }\left\|y_{k}^{*}\right\| \rightarrow 0
$$

one has $\left\|x_{k}^{*}\right\| \rightarrow 0$ as $k \rightarrow \infty$.

Let $\varphi: X \rightarrow \overline{\mathbb{R}}$ be finite at $\bar{x}$. We say that $\varphi$ is sequentially normally epicompact (SNEC) at $\bar{x}$ if its epigraph is (SNC) at $(\bar{x}, \varphi(\bar{x}))$.

Let us recall the definitions of the (local) metric regularity and Lipschitz-like multifunctions introduced by Robinson [24] and Aubin [1], respectively.

Definition 2.1. [16]. Let $\Phi: X \rightrightarrows Y$ be a multifunction between Asplund spaces. Let $(\bar{x}, \bar{y}) \in \operatorname{gph} \Phi$.

(a) $\Phi$ is said to be local-metrically regular around $(\bar{x}, \bar{y})$ with modulus $c>0$ if there exist a neighborhood $U$ of $\bar{x}$, a neighborhood $V$ of $\bar{y}$, and a number $\mu>0$ such that

$$
\operatorname{dist}\left(x, \Phi^{-1}(y)\right) \leq c \operatorname{dist}(y, \Phi(x))
$$

for any $x \in U$ and $y \in V$ satisfying $\operatorname{dist}(y, \Phi(x)) \leq \mu$.

(b) $\Phi$ is said to be pseudo-Lipschitzian or Lipschitz-like around $(\bar{x}, \bar{y})$ with modulus $\ell>0$ if there exist a neighborhood $U$ of $\bar{x}$ and a neighborhood $V$ of $\bar{y}$ such that

$$
\Phi\left(x_{1}\right) \cap V \subset \Phi\left(x_{2}\right)+\ell\left\|x_{1}-x_{2}\right\| B_{X} \quad \forall x_{1}, x_{2} \in U .
$$




\section{Characterizations of Metric Regularity and Lipschitzian Stability}

In this section we establish the sufficient conditions for the metric regularity and the Lipschitzian stability of the implicit multifunction (1.2).

Let $Z$ be an Asplund space and let $\Omega \subset Z$. We say that $\Omega$ has the robustness property at $\bar{z}$ belonging to closure of $\Omega$ if for any sequences $z_{k} \stackrel{\Omega}{\longrightarrow} \bar{z}$ and $z_{k}^{*} \stackrel{w^{*}}{\longrightarrow}$ $z^{*}$ with $z_{k}^{*} \in N\left(z_{k} ; \Omega\right), k=1,2, \ldots$, there exists a subsequence $\left\{z_{k_{j}}^{*}\right\}$ of $\left\{z_{k}^{*}\right\}$ satisfying $z_{k_{j}}^{*} \rightarrow z^{*}$ in the normal topology of $Z^{*}$ and $z^{*} \in N(\bar{z} ; \Omega)$. Obviously, the robustness property of $\Omega$ at $\bar{z}$ automatically holds if $Z$ is a finite-dimensional space.

Let $\Xi: X \rightrightarrows Y$ be a multifunction and let $\left(x_{0}, y_{0}\right) \in$ gph $\Xi$. The Mordukhovich normal coderivative of $\Xi$ is said to be robust at $\left(x_{0}, y_{0}\right)$ if its graph has the robustness property at $\left(x_{0}, y_{0}\right)$.

One says that $\Xi$ is inner semicompact around $x_{0} \in X$ if there exists a neighborhood $U$ of $x_{0}$ such that for any $x \in U$ and any sequence $x_{k} \rightarrow x$, there is a sequence $y_{k} \in \Xi\left(x_{k}\right), k=1,2, \ldots$, which contains a subsequence convergent in the norm topology of $Y$.

Definition 3.1. [24]. For the variational system defined as in (1.1) and (1.2), let $\omega_{0}:=\left(x_{0}, y_{0}, 0\right) \in \operatorname{gph} F$. The implicit multifunction $G$ is said to be localmetrically regular around $\omega_{0}$ with modulus $c>0$ if there exist a neighborhood $U$ of $x_{0}$, a neighborhood $V$ of $y_{0}$ and a number $\mu>0$ such that

$$
\operatorname{dist}(x, G(y)) \leq c \operatorname{dist}(0, F(x, y))
$$

for any $x \in U$ and $y \in V$ satisfying $\operatorname{dist}(0, F(x, y)) \leq \mu$.

Given a subset $\Omega \subset X$ and a point $u \in X$, we denote the set of the metric projections of $u$ on the closure of $\Omega$ by $\mathcal{M}(u, \Omega)$, that is

$$
\mathcal{M}(u, \Omega)=\{x \in \operatorname{cl} \Omega \mid\|x-u\|=\operatorname{dist}(u, \Omega)\}
$$

where dist $(u, \Omega):=\inf _{z \in \Omega}\|z-u\|$ is the distance from $u$ to $\Omega$ and $\operatorname{cl} \Omega$ denotes the closure of $\Omega$.

For the variational system defined as in (1.1) and (1.2), let $\omega_{0}:=\left(x_{0}, y_{0}, 0\right) \in$ gph $F$ and let $F_{y}(\cdot):=F(\cdot, y)$. Consider the following assumption (SC):

There are a neighborhood $U_{0}$ of $x_{0}$, a neighborhood $V_{0}$ of $y_{0}$ such that for any $y \in V_{0}$ and for any $x \in U_{0}$, the multifunction $\mathcal{M}\left(0, F_{y}(\cdot)\right)$ is inner semicompact around $x$.

We first give the pointbased sufficient conditions for the metric regularity and the Lipschitzian stability of implicit multifunction (1.2). 
Theorem 3.2. . Let $X, Y, Z$ be Asplund spaces, a multifunction $F: X \times Y \rightrightarrows Z$ and a multifunction $G: Y \rightrightarrows X$ be defined as in (1.1) and (1.2). Let $\omega_{0}:=$ $\left(x_{0}, y_{0}, 0\right) \in \operatorname{gph} F$. Suppose that gph $F$ is locally closed around $\omega_{0}$ and (SC) is valid. If the Mordukhovich normal coderivative of $F$ has the robustness property at $\omega_{0}$ and the following condition

$$
\forall\left(y^{*}, z^{*}\right) \in Y^{*} \times Z^{*},\left(0, y^{*}\right) \in D^{*} F\left(\omega_{0}\right)\left(z^{*}\right) \Longrightarrow\left(y^{*}, z^{*}\right)=(0,0)
$$

holds, then $G$ is local-metrically regular around $\omega_{0}$ with modulus $\frac{1}{\sigma}>0$, i.e., there exist a neighborhoods $U$ of $x_{0}$, a neighborhood $V$ of $y_{0}$, a number $\sigma>0$ and $a$ number $\mu>0$ such that

$$
\operatorname{dist}(x, G(y)) \leq \frac{1}{\sigma} \operatorname{dist}(0, F(x, y))
$$

for any $x \in U$ and $y \in V$ satisfying $\operatorname{dist}(0, F(x, y)) \leq \mu$. Moreover, for any $x^{*} \in X^{*}$,

$$
D^{*} G\left(y_{0}, x_{0}\right)\left(x^{*}\right) \subset \bigcup_{z^{*} \in Z^{*}}\left\{y^{*}:\left(-x^{*}, y^{*}\right) \in D^{*} F\left(\omega_{0}\right)\left(z^{*}\right)\right\}
$$

and $G$ is Lipschitz-like around $\left(y_{0}, x_{0}\right)$.

For proving Theorem 3.2, we need the following auxiliary results.

We first consider the parametric minimization problem

$$
\min \{\varphi(x, y) \mid y \in \Phi(x)\}
$$

depending on the parameter $x$ and the corresponding marginal function

$$
m(x):=\inf \{\varphi(x, y): y \in \Phi(x)\},
$$

where $\varphi: X \times Y \rightarrow \overline{\mathbb{R}}$ is an extended real-valued function and $\Phi: X \rightrightarrows Y$ is a multifunction between Banach spaces. Let

$$
M(x):=\{y \in \Phi(x) \mid \varphi(x, y)=m(x)\}
$$

denote the parametric solution set of (3.4).

Lemma 3.3. (Basic subgradient of marginal function) [16, Theorem 3.38]. Let $\Phi: X \rightrightarrows Y$ be a closed-graph multifunction between Asplund spaces $X$ and $Y$, let $\varphi: X \times Y \rightarrow \overline{\mathbb{R}}$ be lower semicontinuous on gph $\Phi$. Suppose that for any $\bar{y} \in M(\bar{x})$, the multifunction $M$ in (3.6) be inner semicompact at $(\bar{x}, \bar{y})$ and either $\varphi$ is SNEC at $(\bar{x}, \bar{y})$ or $\operatorname{gph} \Phi$ is SNC at $(\bar{x}, \bar{y})$ and the qualification constraint 


$$
\partial^{\infty} \varphi(\bar{x}, \bar{y}) \cap(-N((\bar{x}, \bar{y}) ; \operatorname{gph} \Phi))=\{0\}
$$

is satisfied. Then one has the inclusion

$$
\partial m(\bar{x}) \subset \bigcup\left\{x^{*}+D^{*} \Phi(\bar{x}, \bar{y})\left(y^{*}\right):\left(x^{*}, y^{*}\right) \in \partial \varphi(\bar{x}, \bar{y}), \bar{y} \in M(\bar{x})\right\} .
$$

Given a single-valued mapping $f: X \rightarrow Y$ between Banach spaces. Let $\bar{x} \in X$. $f$ is said to be locally Lipschitzian around $\bar{x}$ if there exist a neighborhood $U$ of $\bar{x}$ and a number $\ell \geq 0$ such that

$$
\left\|f\left(x_{1}\right)-f\left(x_{2}\right)\right\| \leq \ell\left\|x_{1}-x_{2}\right\| \text { for all } x_{1}, x_{2} \in U .
$$

We recall from [16, Corollary 1.81] that if $X$ is a Banach space and $\varphi: X \rightarrow \overline{\mathbb{R}}$ is locally Lipschitzian around $\bar{x}$, then $\partial^{\infty} \varphi(\bar{x})=\{0\}$.

Lemma 3.4. (Sum rules for basic subgradient) [16, Theorem 3.36]. Let $X$ be an Asplund space, let $\varphi_{i}: X \rightarrow \overline{\mathbb{R}}, i=1,2$, be lower semicontinuous at $\bar{x}$, and one of these functions be SNEC at $\bar{x}$. Suppose that

$$
\left[x_{i}^{*} \in \partial^{\infty} \varphi_{i}(\bar{x}), i=1,2, x_{1}^{*}+x_{2}^{*}=0\right] \Longrightarrow x_{1}^{*}=x_{2}^{*}=0 .
$$

Then one has the inclusion

$$
\partial\left(\varphi_{1}+\varphi_{2}\right)(\bar{x}) \subset \partial \varphi_{1}(\bar{x})+\partial \varphi_{2}(\bar{x}) .
$$

The following result derives a sufficient condition for the metric regularity of $G$ via the partial Mordukhovich normal coderivative of $F$ in $x$.

Theorem 3.5. Let $X, Z$ be Asplund spaces, $Y$ a metric space, $F: X \times Y \rightrightarrows Z$ and $G: Y \rightrightarrows X$ multifunctions are defined as in (1.1) and (1.2), respectively. Let $\omega_{0}:=\left(x_{0}, y_{0}, 0\right) \in \operatorname{gph} F$. Suppose that $\operatorname{gph} F$ is locally closed around $\omega_{0}$ and (SC) is valid. If there exist $\sigma>0$ and neighborhood $U_{0} \times V_{0}$ of $\left(x_{0}, y_{0}\right)$ such that for any $(x, y) \in U_{0} \times V_{0}$ with $0 \notin F(x, y)$ satisfying

$$
\sigma \leq \inf \left\{\left\|x^{*}\right\|: x^{*} \in D^{*} F_{y}(x, z)\left(z^{*}\right), \quad z \in \mathcal{M}\left(0, F_{y}(x)\right),\left\|z^{*}\right\|=1\right\},
$$

then $G$ is metrically regular around $\omega_{0}$ with modulus $\frac{1}{\sigma}>0$, i.e., there exist a neighborhood $U$ of $x_{0}$, a neighborhood $V$ of $y_{0}$ and a number $\mu>0$ such that

$$
\operatorname{dist}(x, G(y)) \leq \frac{1}{\sigma} \operatorname{dist}(0, F(x, y))
$$

for any $x \in U$ and $y \in V$ satisfying $\operatorname{dist}(0, F(x, y)) \leq \mu$. Moreover, if $F$ : $X \times Y \rightrightarrows Z$ is partially Lipschitz-like in $y$ with rank $\ell$ around $\omega_{0}$, i.e., there exist 
a neighborhood $U_{1}$ of $x_{0}$, a neighborhood $V_{1}$ of $y_{0}$ and a neighborhood $W_{0}$ of 0 such that, for any $(x, y) \in U_{1} \times V_{1}$ and $\left(x, y^{\prime}\right) \in U_{1} \times V_{1}$,

$$
F\left(x, y^{\prime}\right) \cap W_{0} \subset F(x, y)+\ell \operatorname{dist}\left(y^{\prime}, y\right) B_{Y},
$$

then $G$ is Lipschitz-like with rank $\frac{\ell}{\sigma}$ around $\left(y_{0}, x_{0}\right)$, i.e., there exist a neighborhood $V$ of $y_{0}$ and a neighborhood $U$ of $x_{0}$ such that

$$
G\left(y^{\prime}\right) \cap U \subset G(y)+\frac{\ell}{\sigma} \operatorname{dist}\left(y^{\prime}, y\right) B_{X} \forall y, y^{\prime} \in V .
$$

Proof. Let $\omega_{0}:=\left(x_{0}, y_{0}, 0\right) \in \operatorname{gph} F$. By our assumptions, there are a neighborhood $U_{0}$ of $x_{0}$ and a neighborhood $V_{0}$ of $y_{0}$ such that (3.9) is valid. Choose a number $\mu>0$ and a number $\rho>0$ such that

$$
\mu<\sigma \rho \text { and } B_{\rho}\left(x_{0}\right) \subset U_{0} .
$$

We now examine the case $(x, y) \in \operatorname{dom} F \cap\left(U_{0} \times V_{0}\right)$ satisfying

$$
\operatorname{dist}(0, F(x, y)) \leq \mu \text {. }
$$

For convenience we will ignore $(x, y) \in \operatorname{dom} F$. Let $U:=U_{0}$ and $V:=V_{0}$. We want to show that $U, V$ together with constants $\mu$ and $\sigma$ satisfy the conclusion of the theorem. Fix any $x \in U$ and $y \in V$ and assume that (3.12) is satisfied. Put $\alpha:=\operatorname{dist}(0, F(x, y))$. By (3.11) and (3.12), $\alpha<\sigma \rho$. It remains to show that

$$
\operatorname{dist}(x, G(y)) \leq \frac{\alpha}{\sigma} .
$$

Consider the function $v_{y}(u):=\operatorname{dist}\left(0, F_{y}(u)\right), u \in U$. We claim that $v_{y}(\cdot)$ is lower semicontinuous on $U$. Indeed, if there is a sequence $x_{k} \rightarrow u$ and $\varepsilon>0$ such that $v_{y}\left(x_{k}\right) \leq v_{y}(u)-\varepsilon$ for every $k \in \mathbb{N}$, then, by (SC), there exist a sequence $\left\{k_{j}\right\} \subset\{k\}$ and a sequence $z_{k_{j}} \in \mathcal{M}\left(0, F_{y}\left(x_{k_{j}}\right)\right)$ such that $z_{k_{j}} \rightarrow z \in Z$. Since $z_{k_{j}} \in F_{y}\left(x_{k_{j}}\right)=F\left(x_{k_{j}}, y\right)$ for every $j \in \mathbb{N}$, the closedness of gph $F$ around $\omega_{0}$ implies $z \in F_{y}(u)$. Hence, from the relation

$$
\left\|z_{k_{j}}\right\|=v_{y}\left(x_{k_{j}}\right) \leq v_{y}(u)-\varepsilon \quad \forall j \in \mathbb{N},
$$

it follows that $v_{y}(u) \leq\|z\| \leq v_{y}(u)-\varepsilon$, which is impossible and our claim is proved. Therefore, $v(\cdot)$ is lower semicontinuous on $B_{\rho}\left(x_{0}\right)$. Fixing any $\delta \in\left(\frac{\alpha}{\rho}, \sigma\right)$, we have $v_{y}(x)=\alpha<\alpha \frac{\sigma}{\delta}$. Putting $t:=\frac{\delta}{\alpha} v_{y}(x)$, we see that

$$
v_{y}(x)=t \frac{\alpha}{\delta}, \quad \text { and } t \in(0, \sigma) .
$$


Clearly, $v_{y}(x) \leq \inf _{u \in B_{\rho}\left(x_{0}\right)} v_{y}(u)+t \frac{\alpha}{\delta}$. From the Ekeland variational principle [16, Theorem 2.26], it follows that there is $\bar{x} \in B_{\rho}\left(x_{0}\right)$ such that

$$
v_{y}(\bar{x}) \leq v_{y}(x), \quad\|\bar{x}-x\| \leq \frac{\alpha}{\delta}
$$

and

$$
v_{y}(\bar{x}) \leq v_{y}(u)+t\|u-\bar{x}\| \quad \forall u \in B_{\rho}\left(x_{0}\right) .
$$

We next claim that

$$
0 \in F_{y}(\bar{x}) .
$$

Conversely, suppose that $0 \notin F_{y}(\bar{x})$. It follows from (3.15) that $\bar{x}$ is a local minimum of the function

$$
\psi(u):=v_{y}(u)+\chi(u), u \in B_{\rho}\left(x_{0}\right),
$$

where $\chi(u):=t\|u-\bar{x}\|$ is a Lipschitz function. From the nonsmooth version of Fermat's rule [16, Proposition 1.114], it follows that

$$
0 \in \partial \psi(\bar{x}) .
$$

By the Lipschitzian property of $\chi$ and Corollary 1.81 in [16], we have $\partial^{\infty} \chi(\bar{x})=$ $\{0\}$ and $\chi$ is sequentially normally epi-compact at $\bar{x}$. From Lemma 3.4, we have

$$
0 \in \partial v_{y}(\bar{x})+t B_{X^{*}}
$$

Let us now compute $\partial v_{y}(\bar{x})$. Define

$$
\begin{aligned}
& \Phi(u):=F_{y}(u), \quad \varphi(u, z):=\|z\|, \\
& m(u):=v_{y}(u)=\inf \{\varphi(u, z): z \in \Phi(u)\} .
\end{aligned}
$$

Take arbitrary $\bar{z} \in M(\bar{x}):=\mathcal{M}\left(0, F_{y}(\bar{x})\right)$. Since $\varphi$ is locally Lipschitzian at $(\bar{x}, \bar{z})$, it is sequentially normally epi-compact at this point and

$$
\partial^{\infty} \varphi(\bar{x}, \bar{z})=\{(0,0)\} .
$$

Define $\theta(z):=\|z\|$. It is easy to check that

$$
\partial \varphi(\bar{x}, \bar{z})=\{0\} \times \partial \theta(\bar{z}) .
$$

Applying Lemma 3.3, we obtain

$$
\partial v_{y}(\bar{x}) \subset \bigcup\left[D^{*} F_{y}(\bar{x}, \bar{z})\left(z^{*}\right) \mid z^{*} \in \partial \theta(\bar{z}), \bar{z} \in \mathcal{M}\left(0, F_{y}(\bar{x})\right)\right] .
$$


The condition $0 \notin F_{y}(\bar{x})$ implies $\bar{z} \neq 0$ for every $\bar{z} \in \mathcal{M}\left(0, F_{y}(\bar{x})\right)$. It follows that

$$
\partial \theta(\bar{z})=\left\{z^{*} \in Z^{*} \mid\left\|z^{*}\right\|=1,\left\langle z^{*}, \bar{z}\right\rangle=\|\bar{z}\|\right\} .
$$

From (3.16)-(3.18), there exist vectors $\bar{z} \in \mathcal{M}\left(0, F_{y}(\bar{x})\right), z^{*} \in Z^{*}$ with $\left\|z^{*}\right\|=1$, and $x^{*} \in D^{*} F_{y}(\bar{x}, \bar{z})\left(z^{*}\right)$ such that $\left\|x^{*}\right\| \leq t<\sigma$. Besides, by (3.9), we can assert that $\sigma \leq\left\|x^{*}\right\|$, this contradicts the inequality $\left\|x^{*}\right\|<\sigma$. We have thus shown that $0 \in F_{y}(\bar{x})$, i.e., $\bar{x} \in G(y)$. Hence, by (3.14),

$$
\operatorname{dist}(x, G(y)) \leq\|x-\bar{x}\| \leq \frac{\alpha}{\delta} .
$$

Letting $\delta \rightarrow \sigma$ we obtain $\operatorname{dist}(x, G(y)) \leq \frac{1}{\sigma} \operatorname{dist}(0, F(x, y))$ and $G$ is localmetrically regular around $\omega_{0}$.

Suppose that $F$ is partially Lipschitz-like in $y$ with rank $\ell$ around $\omega_{0}$. Then there exist a neighborhood $U_{1}$ of $x_{0}$, a neighborhood $V_{1}$ of $y_{0}$ and a neighborhood $W_{0}$ of 0 such that, for any $(x, y),\left(x, y^{\prime}\right) \in U_{1} \times V_{1}$,

$$
F\left(x, y^{\prime}\right) \cap W_{0} \subset F(x, y)+\ell \operatorname{dist}\left(y^{\prime}, y\right) B_{Y} .
$$

Choose $\mu>0$ such that

$$
B_{\mu}(0) \subset W_{0} .
$$

It follows from the regularity of $G$ around $\omega_{0}$ that there exist a neighborhood $U_{2}$ of $x_{0}$, a neighborhood $V_{2}$ of $y_{0}$ and a number $\sigma>0$ such that

$$
\operatorname{dist}(x, G(y)) \leq \frac{1}{\sigma} \operatorname{dist}(0, F(x, y))
$$

for any $x \in U_{2}$ and $y \in V_{2}$ satisfying $\operatorname{dist}(0, F(x, y)) \leq \mu$. Let $U:=U_{1} \cap U_{2}$ and $V:=V_{1} \cap V_{2}$. Then

$$
G\left(y^{\prime}\right) \cap U \subset G(y)+\frac{\ell}{\sigma} \operatorname{dist}\left(y^{\prime}, y\right) B_{X} \forall y, y^{\prime} \in V .
$$

Indeed, take arbitrary $x \in G\left(y^{\prime}\right) \cap U$. From (3.19) and (3.20), it follows that

$$
\operatorname{dist}(x, G(y)) \leq \frac{1}{\sigma} \operatorname{dist}(0, F(x, y)) \leq \frac{\ell}{\sigma} \operatorname{dist}\left(y^{\prime}, y\right) \forall y, y^{\prime} \in V .
$$

Hence, $x \in G(y)+\frac{\ell}{\sigma} \operatorname{dist}\left(y^{\prime}, y\right) B_{X} \forall y, y^{\prime} \in V$. So, (3.21) follows. The proof is complete. 
Remark 3.6. Obviously, (A1) in [10, Theorem 3.1] implies (3.9). The corresponding results for the metric regularity of (1.2) in [10, Theorem 3.2] always require the lower continuity of $F\left(x_{0}, \cdot\right)$ at $y_{0}$ and $F(\cdot)$ is lower semicontinuous at $\left(x_{0}, y_{0}\right)$. Hence, Theorem 3.5 extends Theorem 3.1, Theorem 3.3 in [10] and Theorem 3.4 in [7]. The condition (3.9) is similar to the condition (iv') in [9, Theorem 3.6], but Ledyaev and Zhu [9] assumed that $X$ and $Z$ are Banach spaces with Fréchet-smooth Lipschitzian bump functions, $F\left(x_{0}, \cdot\right)$ is lower semicontinuous at $y_{0}$ and for any fixed $y \in V_{0}, F(\cdot, y)$ is upper semicontinuous.

Lemma 3.7. (Partial coderivative) [16, Corollary 3.17]. Let $F: X \times Y \rightrightarrows Z$ be multifunction between Asplund spaces. Let $\bar{\omega}:=(\bar{x}, \bar{y}, \bar{z}) \in \operatorname{gph} F$ and let $F_{y}(\cdot):=F(\cdot, y)$. Suppose that $\mathrm{gph} F$ is locally closed around $\bar{\omega}$ and PSNC at this point, and that

$$
\left(0, y^{*}\right) \in D_{M F}^{*}(\bar{\omega})(0) \Longrightarrow y^{*}=0 ;
$$

these conditions automatically hold when $F$ is Lipschitz-like around $\bar{\omega}$. Then one has the inclusion

$$
D^{*} F_{\bar{y}}(\bar{x}, \bar{z})\left(z^{*}\right) \subset\left\{x^{*} \in X^{*}: \exists y^{*} \in Y^{*} \text { such that }\left(x^{*}, y^{*}\right) \in D^{*} F(\bar{\omega})\left(z^{*}\right)\right\} .
$$

Next, we give the relationship between the pointbased criteria of $F$ and the partial Mordukhovich normal coderivative of $F$ around a given point.

Theorem 3.8. Let $X, Y, Z$ be Asplund spaces and a multifunction $F: X \times Y \rightrightarrows$ $Z$. Let $\bar{\omega}:=(\bar{x}, \bar{y}, 0) \in \operatorname{gph} F$. Suppose that $\operatorname{gph} F$ is locally closed around $\bar{\omega}$ and its Mordukhovich normal coderivative has the robustness property at this point. Consider the following statements:

(i) For any $\left(y^{*}, z^{*}\right) \in Y^{*} \times Z^{*},\left(0, y^{*}\right) \in D^{*} F(\bar{\omega})\left(z^{*}\right) \Longrightarrow\left(y^{*}, z^{*}\right)=(0,0)$;

(ii) There exist a constant $c>0$, a neighborhood $U$ of $\bar{x}$, a neighborhood $V$ of $\bar{y}$ and a neighborhood $W$ of 0 such that for any point $\omega=(x, y, z) \in$ gph $F \cap(U \times V \times W)$, it holds

$$
\left\|z^{*}\right\| \leq c\left\|x^{*}\right\| \quad \forall z^{*} \in Z^{*}, \forall x^{*} \in D^{*} F_{y}(x, z)\left(z^{*}\right)
$$

(iii) There exist a number $\sigma>0$, a neighborhood $U$ of $\bar{x}$ and a neighborhood $V$ of $\bar{y}$ such that for any $(x, y) \in U \times V$ with $0 \notin F(x, y)$

$$
\sigma \leq \inf \left\{\left\|x^{*}\right\|: x^{*} \in D^{*} F_{y}(x, z)\left(z^{*}\right), z \in \mathcal{M}\left(0, F_{y}(x)\right),\left\|z^{*}\right\|=1\right\} .
$$

Then (i) $\Longrightarrow$ (ii) $\Longrightarrow$ (iii). 
Proof. We first prove that (i) $\Longrightarrow$ (ii). Suppose that (i) holds. We first claim that there exist a neighborhood $U$ of $\bar{x}$, a neighborhood $V$ of $\bar{y}$ and a neighborhood $W$ of 0 such that for any point $\omega=(x, y, z) \in \operatorname{gph} F \cap(U \times V \times W)$ and for any $\left(y^{*}, z^{*}\right) \in Y^{*} \times Z^{*}$ satisfying

$$
\left(0, y^{*}\right) \in D^{*} F(\omega)\left(z^{*}\right) \Longrightarrow\left(y^{*}, z^{*}\right)=(0,0) .
$$

Indeed, if our claim is false, then there exist sequences $\omega_{k}=\left(x_{k}, y_{k}, z_{k}\right) \in \operatorname{gph} F$ and $\left(y_{k}^{*}, z_{k}^{*}\right) \in Y^{*} \times Z^{*} \backslash\{(0,0)\}$ such that for every $k=1,2, \ldots$

$$
\left(0, y_{k}^{*}\right) \in D^{*} F\left(\omega_{k}\right)\left(z_{k}^{*}\right) \text { and } \omega_{k} \rightarrow \bar{\omega} \text { as } k \rightarrow \infty .
$$

Without loss of generality we can assume that $\left\|z_{k}^{*}\right\|=1$ for every $k \in \mathbb{N}$. Consider the following two cases:

Case 1. $\left\{y_{k}^{*}\right\}$ is bounded. Since $Y$ is a Asplund space, the unit ball of the dual space $Y^{*}$ is sequentially weak* compact. Taking into account the boundedness of $\left\{\left(y_{k}^{*}, z_{k}^{*}\right)\right\}$, one may assume that $\left(y_{k}^{*}, z_{k}^{*}\right) \stackrel{w^{*}}{\longrightarrow}\left(y^{*}, z^{*}\right) \in Y^{*} \times Z^{*}$. Clearly,

$$
\left(0, y_{k}^{*},-z_{k}^{*}\right) \in N\left(\omega_{k} ; \operatorname{gph} F\right) .
$$

It follows from the robustness property of Mordukhovich normal coderivative of $F$ at $\omega_{0}$ that $\left(y_{k}^{*}, z_{k}^{*}\right) \rightarrow\left(y^{*}, z^{*}\right)$ as $k \rightarrow \infty$ in the norm topology of $Y^{*} \times Z^{*}$ and $\left(0, y^{*},-z^{*}\right) \in N(\bar{\omega} ; \operatorname{gph} F)$. Then $\left\|z^{*}\right\| \neq 0$ and $\left(0, y^{*}\right) \in D^{*} F(\bar{\omega})\left(z^{*}\right)$ which is a contradiction to (i).

Case 2. $\left\{y_{k}^{*}\right\}$ is not bounded. Then there is a subsequence $\left\{y_{k_{j}}^{*}\right\}$ of $\left\{y_{k}^{*}\right\}$ such that $\left\|y_{k_{j}}^{*}\right\| \rightarrow \infty$ as $j \rightarrow \infty$. Without loss of generality, we may assume that

$$
\left(\frac{y_{k_{j}}^{*}}{\left\|y_{k_{j}}^{*}\right\|}, \frac{z_{k_{j}}^{*}}{\left\|y_{k_{j}}^{*}\right\|}\right) \stackrel{w^{*}}{\longrightarrow}\left(y^{*}, z^{*}\right) \in Y^{*} \times Z^{*} .
$$

Analysis similar to that as in Case 1 shows that $\left\|y^{*}\right\| \neq 0$ and $\left(0, y^{*}\right) \in D^{*} F(\bar{\omega})\left(z^{*}\right)$ which contradicts (i). Hence, our claim is proved. Since the Mordukhovich normal coderivative of $F$ is robust at $\bar{\omega}$, it follows that $F$ is SNC at this point. Combining this with (i) and Theorem 4.10 in [16], we deduce that $F$ is Lipschitz-like around $\bar{\omega}$. By (3.25) and Lemma 3.7, we have

$$
D^{*} F_{y}(x, z)\left(z^{*}\right) \subset\left\{x^{*} \in X^{*}: \exists y^{*} \in Y^{*} \text { such that }\left(x^{*}, y^{*}\right) \in D^{*} F(\omega)\left(z^{*}\right)\right\}
$$

for any $\omega=(x, y, z) \in \operatorname{gph} F \cap(U \times V \times W)$ and $z^{*} \in Z^{*}$.

Our next claim that (ii) holds. On the contrary, suppose that the conclusion of (ii) is not true. Then we can find sequences $\omega_{k}=\left(x_{k}, y_{k}, z_{k}\right) \in \operatorname{gph} F$ and 
$\left(x_{k}^{*}, z_{k}^{*}\right) \in X^{*} \times Z^{*}$ such that $\omega_{k} \rightarrow \bar{\omega}, x_{k}^{*} \in D^{*} F_{y_{k}}\left(x_{k}, z_{k}\right)\left(z_{k}^{*}\right)$, and $\left\|z_{k}^{*}\right\|>k\left\|x_{k}^{*}\right\|$ for all $k \in \mathbb{N}$. There is no loss of generality in assuming that $\left\|z_{k}^{*}\right\|=1$ for all $k \in \mathbb{N}$. Then

$$
x_{k}^{*} \in D^{*} F_{y_{k}}\left(x_{k}, z_{k}\right)\left(z_{k}^{*}\right) \text { and }\left\|x_{k}^{*}\right\| \leq \frac{1}{k} \forall k \in \mathbb{N} .
$$

It follows from (3.27) that there exists a sequence $\left\{y_{k}^{*}\right\} \subset Y^{*}$ such that $\left(x_{k}^{*}, y_{k}^{*}\right) \in$ $D^{*} F\left(\omega_{k}\right)\left(z_{k}^{*}\right)$, where $\omega_{k}:=\left(x_{k}, y_{k}, z_{k}\right)$. Hence

$$
\left(x_{k}^{*}, y_{k}^{*},-z_{k}^{*}\right) \in N\left(\omega_{k} ; \operatorname{gph} F\right) .
$$

If the sequence $\left\{y_{k}^{*}\right\}$ is bounded then, by the same analysis as in Case 1, it follows that there exists a subsequence $\left\{\left(x_{k_{j}}^{*}, y_{k_{j}}^{*}, z_{k_{j}}^{*}\right)\right\}$ of $\left\{\left(x_{k}^{*}, y_{k}^{*}, z_{k}^{*}\right)\right\}$ converging to $\left(0, y^{*}, z^{*}\right)$ in the norm topology of $X^{*} \times Y^{*} \times Z^{*}$ such that $\left\|z^{*}\right\| \neq 0$ and $\left(0, y^{*},-z^{*}\right) \in N(\bar{\omega} ; \operatorname{gph} F)$. Hence $\left\|z^{*}\right\| \neq 0$ and $\left(0, y^{*}\right) \in D^{*} F(\bar{\omega})\left(z^{*}\right)$ which contradicts (i). If the sequence $\left\{y_{k}^{*}\right\}$ is not bounded then, by the same method as in Case 2, it follows that there exists a subsequence $\left\{\left(\frac{x_{k_{j}}^{*}}{\left\|y_{k_{j}}^{*}\right\|}, \frac{y_{k_{j}}^{*}}{\left\|y_{k_{j}}^{*}\right\|}, \frac{z_{k_{j}}^{*}}{\left\|y_{k_{j}}^{*}\right\|}\right)\right\}$ of $\left\{\left(\frac{x_{k}^{*}}{\left\|y_{k}^{*}\right\|}, \frac{y_{k}^{*}}{\left\|y_{k}^{*}\right\|}, \frac{z_{k}^{*}}{\left\|y_{k}^{*}\right\|}\right)\right\}$ converging to $\left(0, y^{*}, z^{*}\right)$ in the norm topology of $X^{*} \times$ $Y^{*} \times Z^{*}$ such that $\left\|y^{*}\right\| \neq 0$ and $\left(0, y^{*}\right) \in D^{*} F(\bar{\omega})\left(z^{*}\right)$ which is a contradiction to (i).

From the above, it follows that there must exist a constant $c>0$, a neighborhood $U$ of $\bar{x}$, a neighborhood $V$ of $\bar{y}$ and a neighborhood $W$ of 0 such that for any point $\omega=(x, y, z) \in \operatorname{gph} F \cap(U \times V \times W)$, (3.23) is fulfilled. The implication $[(\mathrm{ii}) \Longrightarrow(\mathrm{iii})]$ is trivial. The proof is complete.

Proof of Theorem 3.2. Obviously, the local-metric regularity of $G$ at $\omega_{0}$ immediately follows from Theorem 3.5 and Theorem 3.8. Now let us examine the formula (3.3). We first observe that the graph of the mapping $G$ under consideration can be represented as follows

$$
\operatorname{gph} G=\{(y, x) \in Y \times X \mid g(x, y) \in \Theta\} \text { with } \Theta:=\operatorname{gph} F
$$

where $g(x, y):=(x, y, 0)$. Obviously, $g$ is a strictly differentiable function and

$$
\left(\nabla g\left(x_{0}, y_{0}\right)\right)^{*}\left(x^{*}, y^{*}, z^{*}\right)=\left(x^{*}, y^{*}\right) \forall\left(x^{*}, y^{*}, z^{*}\right) \in X^{*} \times Y^{*} \times Z^{*} .
$$

We have

$$
N\left(\omega_{0} ; \Theta\right) \cap \operatorname{ker}\left(\nabla g\left(x_{0}, y_{0}\right)\right)^{*}=\{0\} .
$$


Indeed, let $\left(x^{*}, y^{*}, z^{*}\right) \in N\left(\omega_{0} ; \Theta\right) \cap \operatorname{ker}\left(\nabla g\left(x_{0}, y_{0}\right)\right)^{*}$. Then, by (3.29), $\left(x^{*}, y^{*}\right)=$ $(0,0)$ and $(0,0) \in D^{*} F\left(\omega_{0}\right)\left(z^{*}\right)$. It follows from (PB) that $z^{*}=0$ and (3.30) is fulfilled. Applying Corollary 3.42 in [16] we have

$$
N\left(\omega_{0} ; g^{-1}(\Theta)\right) \subset\left(\nabla g\left(\omega_{0}\right)\right)^{*} N\left(\omega_{0} ; \Theta\right) .
$$

For each $x^{*} \in X^{*}$, let $y^{*} \in D^{*} G\left(y_{0}, x_{0}\right)\left(x^{*}\right)$. Then $\left(y^{*},-x^{*}\right) \in N\left(\left(y_{0}, x_{0}\right)\right.$; gph $\left.G\right)$. It is easy to check that

$$
\left(y^{*},-x^{*}\right) \in N\left(\left(y_{0}, x_{0}\right) ; \operatorname{gph} G\right) \Longleftrightarrow\left(-x^{*}, y^{*}\right) \in N\left(\left(x_{0}, y_{0}\right) ; g^{-1}(\Theta)\right) .
$$

From (3.29) and (3.31) it follows that

$$
\left(-x^{*}, y^{*}\right) \in N\left(\left(x_{0}, y_{0}\right) ; g^{-1}(\Theta)\right) \Longrightarrow \exists z^{*} \in Z^{*},\left(-x^{*}, y^{*}\right) \in D^{*} F\left(\omega_{0}\right)\left(z^{*}\right)
$$

Therefore, (3.3) immediately follows from (3.32) and (3.33). From (3.3) it follows that $D^{*} G\left(y_{0}, x_{0}\right)(0)=\{0\}$. Since the Mordukhovich normal coderivative of $F$ is robust at $\omega_{0}$, it follows that it is $\mathrm{SNC}$ at $\omega_{0}$. By Theorem 3.84 in $[16], g^{-1}(\Theta)$ is $\mathrm{SNC}$ at $\left(x_{0}, y_{0}\right)$ (note that, by the special structure of $g$ as above, we see check at once that Theorem 3.84 in [16] is still fulfilled with the local closedness of $\Theta$ around $\left.\omega_{0}\right)$. Since $\operatorname{gph} F$ is locally closed around $\omega_{0}$ it follows that $\operatorname{gph} G$ is locally closed around $\left(y_{0}, x_{0}\right)$. Applying Theorem 4.10 in [16] we conclude that $G$ is Lipschitz-like around $\left(y_{0}, x_{0}\right)$. The proof is complete.

The following corollary extends the corresponding result in [27].

Corollary 3.9. Let $F: \mathbb{R}^{m} \times \mathbb{R}^{n} \rightrightarrows \mathbb{R}^{p}$ be a multifunction and $G: \mathbb{R}^{n} \rightrightarrows \mathbb{R}^{m} a$ multifunction defined as in (1.1) and (1.2). Let $\omega_{0}:=\left(x_{0}, y_{0}, 0\right) \in \operatorname{gph} F$. Suppose that $\mathrm{gph} F$ is closed around $\omega_{0}$. If the following pointbased condition

$$
\forall\left(y^{*}, z^{*}\right) \in R^{n} \times R^{p},\left(0, y^{*}\right) \in D^{*} F\left(\omega_{0}\right)\left(z^{*}\right) \Longrightarrow\left(y^{*}, z^{*}\right)=(0,0)
$$

holds, then $G$ is local-metrically regular around $\omega_{0}$ and Lipschitz-like around $\left(y_{0}, x_{0}\right)$.

Proof. The proof is immediate from Theorem 3.2, so can be omitted.

\section{Applications to Stability Analysis for Solution Mapping of Parametric Generalized Equations}

In this section we consider a special case of $F$ in (1.1) which has the form $F(x, y)=f(x, y)+Q(x, y)$ where $f: X \times Y \rightarrow Z$ is a single-valued mapping 
and $Q: X \times Y \rightrightarrows Z$ is a set-valued mapping between Asplund spaces. Then (1.1) becomes

$$
0 \in f(x, y)+Q(x, y)
$$

This generalized equation was introduced by Robinson [24]. It is well known that model (4.1) provides a convenient framework for the unified study of optimal solutions in many optimization-related areas including mathematical programming, complementarity, variational inequalities, optimal control, mathematical economics, equilibrium, etc.; see, e.g., $[16,17,25]$ and the references therein for more information and discussions. When $Q(x, y)=N(y ; \Omega)$ the normal cone operator for a convex set $\Omega$, the parametric generalized equations (4.1) is reduced to the parametric variational inequality:

$$
\text { Find } y \in \Omega \text { such that }\langle f(x, y), z-y\rangle \geq 0 \forall z \in \Omega,
$$

which is of particular interest for applications.

The solution map $G: Y \rightrightarrows X$ associated with (4.1) is defined by

$$
G(y)=\{x \in X: 0 \in f(x, y)+Q(x, y)\} .
$$

For the variational system defined as in (4.1) and (4.3), let $\omega_{0}:=\left(x_{0}, y_{0},-f\left(x_{0}\right.\right.$, $\left.\left.y_{0}\right)\right) \in \operatorname{gph} Q$ and let $Q_{y}(\cdot):=Q(\cdot, y)$. Consider the following assumption (SC'):

There are a neighborhood $U_{0}$ of $x_{0}$, a neighborhood $V_{0}$ of $y_{0}$ such that for any $y \in V_{0}$ and for any $x \in U_{0}$, the multifunction $\mathcal{M}\left(-f(\cdot, y), Q_{y}(\cdot)\right)$ is inner semicompact around $x$.

In what follows we establish the sufficient conditions ensuring the metric regularity and Lipschitzian stability for parametric generalized equation (4.3).

Theorem 4.1. Let $X, Y, Z$ be Asplund spaces. Let $f: X \times Y \rightarrow Z$ be a single-valued mapping, $Q: X \times Y \rightrightarrows Z$ and $G: Y \rightrightarrows X$ multifunctions defined as in (4.1) and (4.3). Let $\omega_{0}:=\left(x_{0}, y_{0},-f\left(x_{0}, y_{0}\right)\right) \in \operatorname{gph} Q$. Suppose that $f$ is strictly differentiable at $\left(x_{0}, y_{0}\right), \operatorname{gph} Q$ is locally closed around $\omega_{0}$ and (SC') is valid. If the Mordukhovich normal coderivative of $Q$ has the robustness property at $\omega_{0}$ and for each $\left(y^{*}, z^{*}\right) \in Y^{*} \times Z^{*}$, the following condition holds

$\left(-\nabla f_{x}\left(x_{0}, y_{0}\right)^{*}\left(z^{*}\right), y^{*}-\nabla f_{y}\left(x_{0}, y_{0}\right)^{*}\left(z^{*}\right)\right) \in D^{*} Q\left(\omega_{0}\right)\left(z^{*}\right) \Longrightarrow\left(y^{*}, z^{*}\right)=(0,0)$

then $G$ is local-metrically regular around $\omega_{0}$ with modulus $\frac{1}{\sigma}>0$, i.e., there exist a neighborhoods $U$ of $x_{0}$, a neighborhood $V$ of $y_{0}$, a number $\sigma>0$ and a number $\mu>0$ such that

$$
\operatorname{dist}(x, G(y)) \leq \frac{1}{\sigma} \operatorname{dist}(-f(x, y), Q(x, y))
$$


for any $x \in U$ and $y \in V$ satisfying dist $(-f(x, y), Q(x, y)) \leq \mu$. Moreover, for any $x^{*} \in X^{*}$,

$$
\begin{aligned}
D^{*} G\left(y_{0}, x_{0}\right)\left(x^{*}\right) \subset & \bigcup_{z^{*} \in Z^{*}}\left\{y^{*}:\left(-x^{*}-\nabla f_{x}\left(x_{0}, y_{0}\right)^{*}\left(z^{*}\right), y^{*}\right.\right. \\
& \left.\left.-\nabla f_{y}\left(x_{0}, y_{0}\right)^{*}\left(z^{*}\right)\right) \in D^{*} Q\left(\omega_{0}\right)\left(z^{*}\right)\right\}
\end{aligned}
$$

and $G$ is Lipschitz-like around $\left(y_{0}, x_{0}\right)$.

Proof. Let $F(x, y)=f(x, y)+Q(x, y),(x, y) \in X \times Y$. Since $f$ is strictly differentiable at $\left(x_{0}, y_{0}\right)$ and $\operatorname{gph} Q$ is locally closed around $\omega_{0}$, it follows from Theorem 3.5 in [20] that

$$
D^{*} F\left(x_{0}, y_{0}, 0\right)\left(z^{*}\right)=\nabla f\left(x_{0}, y_{0}\right)^{*}\left(z^{*}\right)+D^{*} Q\left(\omega_{0}\right)\left(z^{*}\right) \quad \forall z^{*} \in Z^{*} .
$$

Hence, the Mordukhovich normal coderivative of $F$ has the robustness property at $\left(x_{0}, y_{0}, 0\right)$. Clearly, gph $F$ is locally closed around $\left(x_{0}, y_{0}, 0\right)$. From (4.4) and (4.7) it follows that

$$
\forall\left(y^{*}, z^{*}\right) \in Y^{*} \times Z^{*}, \quad\left(0, y^{*}\right) \in D^{*} F\left(x_{0}, y_{0}, 0\right)\left(z^{*}\right) \Longrightarrow\left(y^{*}, z^{*}\right)=(0,0) .
$$

Applying Theorem 3.2 we can assert that $G$ is both local-metrically regular around $\omega_{0}$ and Lipschitz-like around $\left(y_{0}, x_{0}\right)$, and (4.6) is fulfilled. The proof is complete.

The following corollary follows immediately from Theorem 4.1.

Corollary 4.2. Let $f: \mathbb{R}^{m} \times \mathbb{R}^{n} \rightarrow \mathbb{R}^{p}$ be a single-valued mapping, $Q: \mathbb{R}^{m} \times \mathbb{R}^{n}$ $\rightrightarrows \mathbb{R}^{p}$ and $G: \mathbb{R}^{n} \rightrightarrows \mathbb{R}^{m}$ multifunctions defined as in (4.1) and (4.3). Let $\omega_{0}:=$ $\left(x_{0}, y_{0},-f\left(x_{0}, y_{0}\right)\right) \in \operatorname{gph} Q$. Suppose that $f$ is strictly differentiable at $\left(x_{0}, y_{0}\right)$ and $\operatorname{gph} Q$ is closed around $\omega_{0}$. If for each $\left(y^{*}, z^{*}\right) \in \mathbb{R}^{n} \times \mathbb{R}^{p}$ the following condition holds

$\left(-\nabla f_{x}\left(x_{0}, y_{0}\right)^{*}\left(z^{*}\right), y^{*}-\nabla f_{y}\left(x_{0}, y_{0}\right)^{*}\left(z^{*}\right)\right) \in D^{*} Q\left(\omega_{0}\right)\left(z^{*}\right) \Longrightarrow\left(y^{*}, z^{*}\right)=(0,0)$, then $G$ is both local-metrically regular around $\omega_{0}$ and Lipschitz-like around $\left(y_{0}, x_{0}\right)$.

\section{REFERENCES}

1. J.-P. Aubin, Lipschitz behavior of solutions to convex minimization problems, Math. Oper. Res., 9 (1984), 87-111.

2. D. Azé, A unified theory for metric regularity of multifunctions, J. Convex Anal., 13 (2006), 225-252.

3. J. M. Borwein and D. M. Zhuang, Verifiable necessary and sufficient conditions for regularity of set-valued and single-valued maps, J. Math. Anal. Appl., 134 (1988) 441-459. 
4. A. L. Dontchev and A. S. Lewis, Aubin criterion for metric regularity, Set-Valued Anal., 13 (2005), 417-438.

5. A. L. Dontchev, M. Quicampoix and N. Zlateva, Aubin criterion for metric regularity, J. Convex Anal., 13 (2006), 281-297.

6. A. L. Dontchev and R. T. Rockafellar, Robinson's implicit function theorem and its extensions, Math. Program., 117 (2009), Ser. B, 129-147.

7. N. Q. Huy and J.-C. Yao, Metric regularity of parametric generalized inequality systems, submitted.

8. V. Jeyakumar and N. D. Yen, Solution stability of nonsmooth continuous systems with applications to cone-constrained optimization, SIAM J. Optim., 14 (2004), 1106-1127

9. Yu. S. Ledyaev and Q. J. Zhu, Implicit multifunctions theorems, Set-Valued Anal., 7 (1999), 209-238.

10. G. M. Lee, N. N. Tam and N. D. Yen, Normal coderivative for multifunctions and implicit function theorems, J. Math. Anal. Appl., 338 (2008), 11-22.

11. B. S. Mordukhovich, Complete characterization of openness, metric regularity, and Lipschitzian properties of multifunctions, Trans. Amer. Math. Soc., 340 (1993), 1-35.

12. B. S. Mordukhovich, Lipschitzian stability of constraint systems and generalized equations, Nonlinear Anal., 22 (1994), 173-206.

13. B. S. Mordukhovich and Y. Shao, Stability of set-valued mappings in infinite dimensions: point criteria and applications, SIAM J. Control Optim., 35 (1997), 285-314.

14. B. S. Mordukhovich, Coderivative analysis of variational systems, J. Global Optimi., 28 (2004), 347-362.

15. B. S. Mordukhovich, Coderivative calculus and robust Lipschitzian stability for variational systems, J. Convex Anal., 13 (2006), 799-822.

16. B. S. Mordukhovich, Variational Analysis and Generalized Differentiation, Vol. I: Basic Theory, Springer, Berlin, 2006.

17. B. S. Mordukhovich, Variational Analysis and Generalized Differentiation, Vol. II: Applications, Springer, Berlin, 2006.

18. B. S. Mordukhovich and Y. Shao, Differential characterizations of covering, metric regularity, and Lipschitzian properties of multifunctions between Banach spaces, Nonlinear Anal., 25 (1995), 1401-1424.

19. B. S. Mordukhovich and Y. Shao, Nonsmooth sequential analysis in Asplund spaces, Trans. Amer. Math. Soc., 348 (1996), 1235-1280.

20. B. S. Mordukhovich and Y. Shao, Nonconvex differential calculus for infinite-dimensional multifunctions, Set-Valued Anal., 4 (1996), 205-236.

21. B. S. Mordukhovich, N. M. Nam and N. D. Yen, Subgradients of maginal functions in parametric mathematical programming, Math. Program., 116 (2009), Ser B., 369396. 
22. H. V. Ngai and M. Théra, Error bounds and implicit multifunction theorem in smooth Banach spaces and applications to optimization, Set-Valued Anal., 12 (2004), 195223.

23. J.-P. Penot, Metric regularity, openess, and Lipschitzian behavior of multifunctions, Nonlinear Anal., 13 (1989), 629-643.

24. S. M. Robinson, Stability theory for systems of inequalities, II. Differentiable nonlinear systems, SIAM J. Numer. Anal., 13 (1976), 497-513.

25. R. T. Rockafellar and R. J.-B. Wets, Variational Analysis, Springer, Berlin, 1998.

26. N. D. Yen, Implicit function theorems for set-valued maps, Acta Math. Vietnam, 12 (1987), no. 2, 17-28.

27. N. D. Yen and J.-C. Yao, Pointbased sufficient conditions for metric regularity of implicit multifunctions, Nonlinear Analysis, TMA, 2009 (to appear).

\author{
N. Q. Huy \\ Department of Applied Mathematics, \\ National Sun Yat-sen University, \\ Kaohsiung 804, Taiwan \\ and \\ Department of Mathematics, \\ Hanoi Pedagogical University, \\ No. 2, Vinh Phuc, \\ Vietnam \\ E-mail: huyngq@yahoo.com \\ J.-C. Yao \\ Department of Applied Mathematics, \\ National Sun Yat-Sen University, \\ Kaohsiung 804, Taiwan \\ E-email: yaojc@math.nsysu.edu.tw
}

